\title{
Erosion Band Features for Cell Phone Image Based Plant Disease Classification
}

\author{
Marion Neumann*, Lisa Hallau**, Benjamin Klatt ${ }^{\ddagger}$, Kristian Kersting $\| \S$ and Christian Bauckhage ${ }^{\S *}$ \\ *Bonn-Aachen International Center for Information Technology, University of Bonn, Germany \\ Email: marion.neumann@uni-bonn.de, \\ ${ }^{* *}$ Institute for Crop Science and Resource Conservation (INRES) - Phytomedicine, University of Bonn, Germany \\ Email: hallau@uni-bonn.de \\ $\ddagger$ Central Institute for Decision Support Systems in Crop Protection (ZEPP), Bad Kreuznach, Germany \\ Email: info@zepp.info \\ $\|_{\text {Computer Science Department, Technical University of Dortmund, Germany }}$ \\ Email: kristian.kersting@cs.tu-dortmund.de \\ $\S$ Fraunhofer IAIS, Sankt Augustin, Germany \\ Email: christian.bauckhage@iais.fraunhofer.de
}

\begin{abstract}
We introduce a novel set of features for a challenging image analysis task in agriculture where cell phone camera images of beet leaves are analyzed as to the presence of plant diseases. Aiming at minimal computational costs on the cellular device and highly accurate prediction results, we present an efficient detector of potential disease regions and a robust classification method based on texture features. We evaluate several first- and second-order statistical features for classifying textures of leaf spots and we find that a combination of descriptors derived on multiple erosion bands of the RGB color channels, as well as, the local binary patterns of gradient magnitudes of the extracted regions accurately distinguish between symptoms caused by five diseases, including infections of the fungi Cercospora beticola, Ramularia beticola, Uromyces betae, and Phoma betae, and the bacterium Pseudomonas syringae pv. aptata.
\end{abstract}

\section{INTRODUCTION}

Mobile devices such as smart phones have become widespread consumer products and the prevalence and ease of use of smart phone cameras provides new challenges and opportunities for image processing and analysis. Opportunities arise from the availability of digital cameras in environments where they were not naturally present only a few years ago. Challenges are due to the fact that implementations of image processing algorithms on a cell phone have to comply with particular characteristics such as constrained battery life, restricted computational power, or limited bandwidth. The work reported here results from a project on using cell phone images in an agricultural scenario. We are developing a system where farmers take pictures of plants they suspect to be infected by a disease such as shown in Figure 1(a). Information extracted therefrom are then send to a central server and analysis results are supposed to be reported back to the farmer while still in the field. In this setting, efficient and reliable image analysis is pivotal. Given the weak connection strengths out in the fields or the increased fees for high volume data transfer, it is hardly possible to transmit several pictures of sufficient resolution. If, on the other hand, the extraction of regions of interest or even the feature computation were performed by an

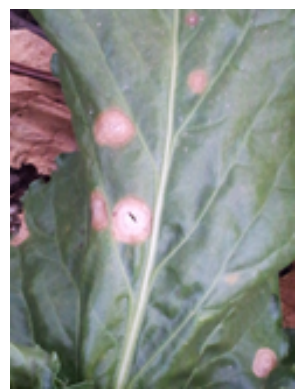

(a) cell phone image

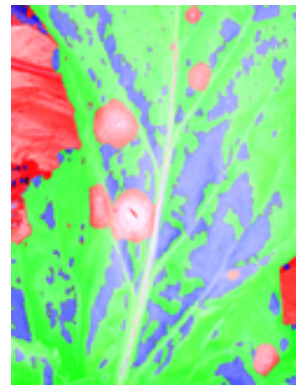

(b) color filter

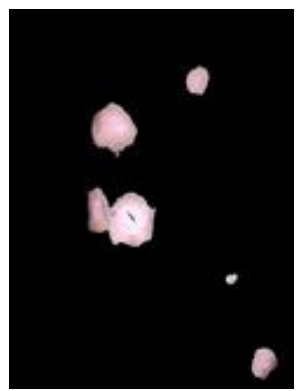

(c) extracted regions
Fig. 1. Cell phone camera image of a beet leaf suffering from Phoma betae (a), and preprocessing steps: color filter (b) and extracted regions (c).

app running on the cell phone, transmission times and costs reduce considerable. In this case, however, elaborate image processing techniques being both robust and limited to the restricted computational resources need to be applied.

Addressing these issues, we present a cascade of efficient image preprocessing, feature extraction, and classification steps tailored to the recognition of pathogens that infect beet plants [1]. Beet is a widely cultivated commercial crop used, e.g., to produce table sugar but fungal and bacterial attacks frequently reduce yields. An early recognition of disease onsets assisted by our system may limit the amount of fungicides needed for pest control and can thus reduce costs and environmental burden. In particular, we attempt to automatically recognize symptoms of five common kinds of infections:

Cercospora beticola (cf. Fig. 2(a)) is a fungal plant pathogen. Infected beet plants show leaf spots that are round blemishes with a definite edge between infected and healthy leaf tissue; while the border of a spot is typically darker and of brownishreddish hue, spot interiors appear bright with dark spores.

Ramularia beticola (cf. Fig. 2(b)), also a fungal pathogen infecting beet plants, shows irregular leaf spots with light 


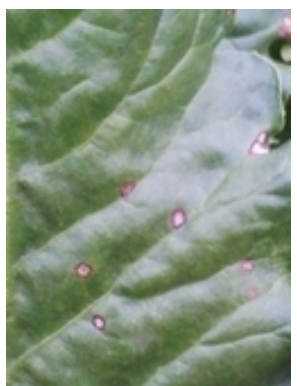

(a) Cercospora

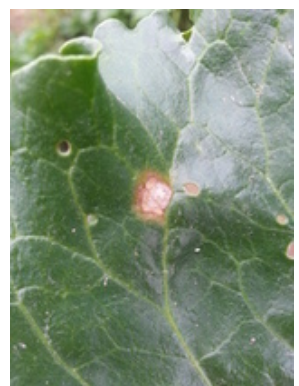

(b) Ramularia

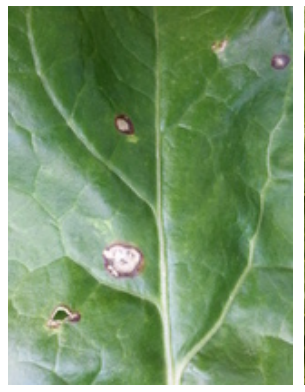

(c) Pseudomonas

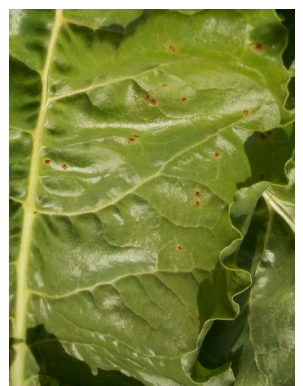

(d) Rust

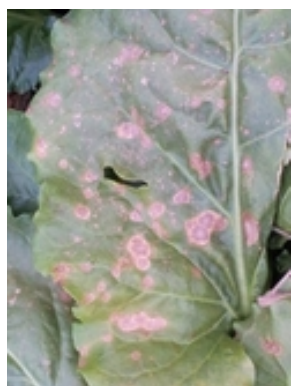

(e) Phoma

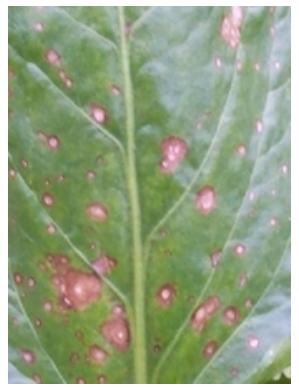

(f) Cercospora \& Phoma

Fig. 2. Cell phone camera images of beet leaves showing leaf spots caused by (a) Cercospora beticola (cerc), (b) Ramularia beticola (ram), (c) Pseudomonas syringae (pseu), (d) Uromyces betae (rust), (e) Phoma betae (phom), and (f) combined infestation of Cercospora and Phoma.

interior surrounded by a light brown to brown border. The interior shows white spores. Leaf spots at a pronounced stage are likely to chap or form coadunate regions.

Pseudomonas syringae pv. aptata (cf. Fig. 2(c)), the only considered bacterial pathogen commonly infecting beets, shows light to dark brown spots potentially with a light interior. The spots may have a brown to black edge.

Uromyces betae (cf. Fig. 2(d)) is a fungal pathogen causing sugar beet rust. Typical symptoms are small reddish-brownish spots surrounded by yellow areas.

Phoma betae (cf. Fig. 2(e)) is a soil-borne fungus and plant pathogen showing rather large leaf spots of concentric rings with irregular shape and less pronounced edges; borders of a spot are yellowish or light brown, spot interiors are characterized by darker brownish hues; when several spots are present, they can grow together and form larger blotches.

Similar to existing approaches to plant disease classification as described in [2] and [3], we rely on texture descriptors [4] for efficient and accurate classification. In contrast to these existing approaches, however, we leverage an ensemble of local features reflecting the spatial structure of the considered leaf spots. This is necessary because we aim to classify early stage symptoms where the leaf spots caused by the considered diseases have a very similar appearance making them even indistinguishable by human experts. Further, data processing is performed on a cellular device requiring resource constraint computations and the consulting system should provide advice in an online fashion while the farmer is still in field. The issues arising in such a scenario covering efficient region detection and feature computation on the smart phone followed by reliable disease classification have not been addressed in previous approaches.

To deal with changes in illumination, scale and perspective changes we take a statistical approach using field data reflecting the variety of the real environment. The data considered for learning and evaluation are cell phone images from unconstrained settings - different camera types, different resolutions, no constraints on how to take the image. Based on the appearance of the infection symptoms, we develop discriminant features by applying multiple erosion steps to the extracted regions and computing several first- and secondorder statistical texture features on the RGB color channels, as well as on the local binary patterns (LBPs) of the gradient magnitude values [5]. The resulting erosion feature ensemble (EFE) proves superior classification performance, in comparison to solely applying the same texture descriptors to the whole image regions, leading to disease detection rates of $95 \%$ and classification accuracies of $84 \%$ in a challenging 6 -class classification problem. Erosion based texture descriptors are well justified by the phenotype of the disease symptoms and go beyond known approaches to plant disease classification.

The next section details the image processing steps in our approach and introduces our newly developed erosion band features for leaf spot classification. In Section III, we present an extensive feature study comparing a wide range of texture features computed on the proposed erosion bands and we evaluate the best performing feature combination on a comprehensive dataset of 2957 regions extracted from 495 images recorded with six different cell phone camera types.

\section{IMAGE PROCESSING}

Our proposed classification method essentially consists of three steps: region detection, feature extraction and class prediction. Whereas the region extraction is applied to the whole input image, feature computation and classification is performed on each extracted region. This approach enables us to identify leaves showing infection symptoms from multiple diseases as illustrated in Figure 2(f). In the following, we give a detailed description of each step in our approach.

\section{A. Region Detection}

The images that are to be analyzed in our scenario are recorded under uncontrollable conditions. Whenever farmers in the field take snapshots of plant leaves, scene illumination, camera angle, focal length, and distance to object are essentially random variables (cf. Figure 2). In order to be able to circumvent diminishing effects due to some of these variables, the default setting of our system is to consider rather high resolution images. Given an RGB image $I$ of 5 resp. 8 megapixels in JPG format, the following preprocessing steps yielded useful intermediate representations for later analysis: 1.) down-scale the input image $I$ by $25 \%$; the resulting image $D$ is of size $484 \times 648 \times 3$ resp. $612 \times 816 \times 3$ depending on the used smart phone camera; this increases sharpness and facilitates further computation; cf. Figure 1(a).

2.) compute a binary image $B$ from $D$ such that the foreground pixels are the pixels with a red value higher than both the 


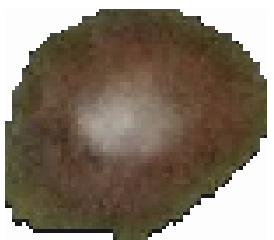

(a) RGB image

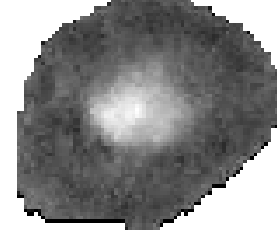

(b) intensity image

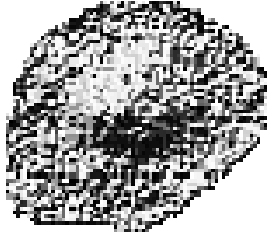

(c) intensity LBP image

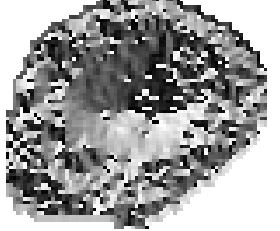

(d) gradient direction image

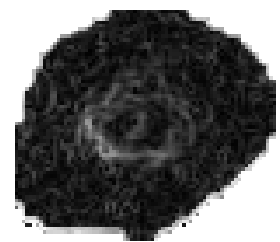

(e) gradient magnitude image

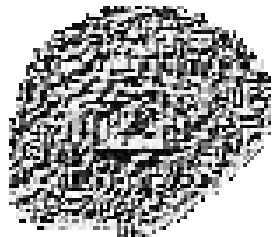

(f) gradient magnitude LBP image

Fig. 3. (a) RGB image, (b) and (c) intensity-based images, and (d)-(f) gradient-based images of an example Cercospora region.

green and the blue value; this pronounces reddish/brownish image regions which may hint at leaf spots caused by fungus or bacteria; Figure 1(b) shows the maximal color values in $D$. 3.) compute a region image $R$ from $B$; this subsumes median filtering, connected component analysis, hole filling, filtering of regions adjacent to the image borders, as well as filtering of regions where the width/height ratio of their bounding boxes is not in the interval $[0.7,2]$; the latter is motivated from biological expertise: since leaf spots tend to be compact, too elongated regions can be discarded from further analysis.

4.) suppress all pixels in $D$ that are background pixels in $R$; the information in the new image $G$ forms the basis for the subsequent analysis; an image $G$ is shown in Figure 1(c).

Note that up to this point none of the steps in our processing cascade makes use of floating point operations. As each of these intermediate steps can be computed using only integer arithmetics, they admit implementations even on older generation phones without processors with floating point units.

\section{B. Statistical Texture Features}

Feature computation and further classification is performed on each detected region separately. A region $r_{i}$ is defined as a connected component in $R$. Each $r_{i}$ is scaled such that its larger image dimension measures 75 pixels. As we are not assuming a particular stage of infection, the leaf spots are typically indistinguishable by their forms and sizes. Hence, established shape descriptors and shape recognition methods, such as form factors [6], bounding box signatures [7], or entropies of curves [8], [9] lack of discriminative power for the classification task. We therefore resort to texture descriptors.

As the main characteristics of the leaf spots caused by the considered diseases illustrated in Figure 2 are based on color values and intensity changes, we analyse color-, intensity-, and gradient-based features. From the original RGB values of each $r_{i}$ we compute the following values (cf. Fig. 3):

- $\quad$ red (R), green (G), blue (B) channels,

- intensities (INT),

- LBPs of intensities (INT LBP),

- gradient magnitudes $\left(\mathrm{G}_{\mathrm{mag}}\right)$,

- $\quad$ gradient directions $\left(\mathrm{G}_{\mathrm{dir}}\right)$, and

- $\quad$ LBPs of gradient magnitudes $\left(\mathrm{G}_{\mathrm{mag}} \mathrm{LBP}\right)$,

where LBP is the basic local binary pattern defined on an 8neighborhood. The LBP value for a pixel $p$ is given by

$$
L B P(p)=\sum_{n=0}^{7} s\left(q_{n}-p\right) 2^{n}
$$

where $q_{n}$ is the $n$-th neighbour of pixel $p$ and $s(x)=1$ for $x \geq 1$ and $s(x)=0$ otherwise. LBP descriptors [5] provide an efficient way to characterize local textures and there are numerous accounts in the recent literature where they were reported to enable highly accurate classification [10], [11].

To analyse the color and gradient information of the extracted regions, we consider simple and co-occurrence based statistical texture features.

First-order statistics computed from the respective normalized histogram $h_{i}$ of $n$ bins lead to simple characteristics of image texture. We consider:

$$
\begin{aligned}
& \text { - MEAN: } \sum_{k=1}^{n} k h_{i k}=\mu_{i} \\
& \text { - VARIANCE: } \sum_{k=1}^{n} h_{i k}\left(k-\mu_{i}\right)^{2} \\
& \text { - ENTROPY: }-\sum_{k=1}^{n} h_{i k} \log \left(h_{i k}\right)
\end{aligned}
$$

and set $n=256$ for all computations.

Second-order statistics computed from the spatial cooccurrence of pixel values analyse relationships among pairs of pixels and therefore constitute more powerful texture descriptors [4]. We consider seven statistics on the $m \times m$ cooccurrence matrix $C(\theta, d)$, where $\theta \in\left\{0^{\circ}, 45^{\circ}, 90^{\circ}, 135^{\circ}\right\}$ defines the angle and $d \in\{1,3,5\}$ defines the distance of the pixel relation. Let the mean $\mu=\frac{1}{m^{2}} \sum_{i, j} C(i, j)$, the mean of the row and column sums $\mu_{i}=\frac{1}{m} \sum_{i} i \sum_{j} C(i, j)$ and $\mu_{j}=\frac{1}{m} \sum_{j} j \sum_{i} C(i, j)$, and the standard deviation of the row and column sums $\sigma_{i}=\frac{1}{m} \sum_{i}\left(i-\mu_{i}\right)^{2} \sum_{j} C(i, j)$ and $\sigma_{j}=\frac{1}{m} \sum_{j}\left(j-\mu_{j}\right)^{2} \sum_{i} C(i, j)$. The second-order statistics are

$$
\begin{aligned}
& \text { - CORRELATION: } \sum_{i, j} \frac{\left(i-\mu_{i}\right)\left(j-\mu_{j}\right) C(i, j)}{\sigma_{i} \sigma_{j}} \\
& \text { - } \quad \text { ENTROPY: }-\sum_{i, j} C(i, j) \log C(i, j) \\
& \text { - INVERSE DIFFERENCE MOMENT: } \sum_{i, j} \frac{1}{1+(i-j)^{2}} C(i, j) \\
& \text { - VARIANCE: } \sum_{i, j} C(i, j)(i-\mu)^{2}
\end{aligned}
$$

where $\mu$ is the mean, $\mu_{i}$ and $\mu_{j}$ resp. $\sigma_{i}$ and $\sigma_{j}$ are the means resp. standard deviations of row and column sums, and

$$
\text { - } \quad \text { SUM ENTROPY: }-\sum_{k=1}^{2 m-1} p_{+}(k) \log p_{+}(k)=s e
$$



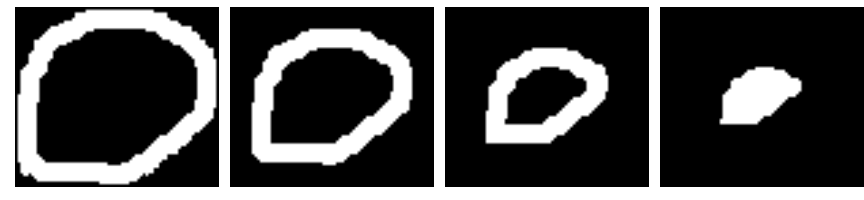

(a) erosion bands
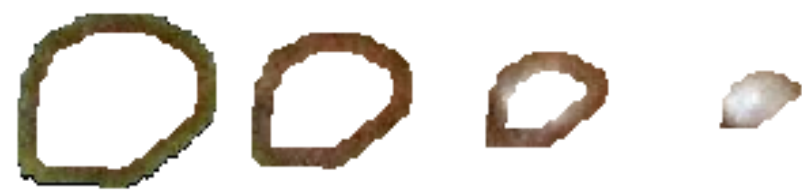

(b) masked RBG regions

Fig. 4. Erosion of the example Cercospora region shown in Figure 3(a).

- DIFFERENCE ENTROPY: $-\sum_{k=1}^{m} p_{-}(k) \log p_{-}(k)$

- SUM VARIANCE: $\sum_{k=1}^{2 m-1}(k-s e)^{2} p_{+}(k)$

where $p_{+}(k)=\sum_{i, j} C(i, j)$ with $k=i+j$ and $p_{-}(k)=$ $\sum_{i, j} C(i, j)$ with $k=|i-j|$. To achieve rotation invariance, each feature value is an average of the statistics computed from $C(\theta, d)$ over the angles $\theta$. The resulting texture descriptor (TEXTURE) is a 7-dimensional feature vector only depending on the distance $d$. We also tested ANGULAR SECOND MOMENT and DIFFERENCE VARIANCE, however, adding these features to the descriptor decreased classification performance in an initial set of experiments on the training data. The considered features covering gradient- and LBP-based texture descriptors considerably extend those suggested in previous work [2], [3].

\section{Erosion Band Features}

Following experts' descriptions of the spots on beet leaves caused by the considered diseases, we propose erosion band features for disease classification. Similar to erosion band signatures [12], we want to extract more fine grained texture features following the circular structure of the leaf spots. Given the erosion width $w$, we iteratively apply binary erosion to the extracted region $r_{i}$. Given the extracted region $r_{i}=r_{i}^{(0)}$ and $E_{w}$ a spherical structuring element, the erosion band at the $t$-th erosion step $b_{i}^{(t)}$ is computed as

$$
b_{i}^{(t)}=r_{i}^{(t-1)}-r_{i}^{(t)} \text { with } r_{i}^{(t)}=r_{i}^{(t-1)} \ominus E_{w},
$$

where $\ominus$ is the morphological erosion operator and $t=$ $\left[1, \ldots, t_{\max }\right]$. The erosion width can be deduced from a given number of iterations $t_{\max }$ and the region dimensions. Figure 4 shows the erosion masks and eroded RBG regions of the Cercospora region depicted in Figure 3 using four erosion steps. For each erosion step we compute the desired texture descriptors on the respective values of the masked regions and concatenate the features. By limiting $t_{\max }$ and filling the erosion band $b_{i}^{\left(t_{\max }\right)}$ in the last iteration, we ensure a fixed feature dimension across all regions. We set $t_{\max }=4$ in all experimental evaluations. Using less erosion steps produces less discriminant features, whereas setting $t_{\max }>4$ unnecessarily increases the feature dimensionality. Note, that the dimension of an erosion band feature is $t_{\max }$ times larger than the dimension of the according feature computed on
TABLE I. NUMBER OF REGIONS PER CLASS FOR THE TWO CONSIDERED DATASETS STUDY AND FULL.

\begin{tabular}{crrrrrrr}
\hline & \multicolumn{7}{c}{ classes } \\
\cline { 2 - 8 } dataset & $n$-inf & cerc & rust & pseu & ram & phom & total \\
\hline STUDY & 55 & 57 & 47 & 44 & 57 & 36 & 296 \\
FULL & 1105 & 1006 & 72 & 494 & 255 & 55 & 2957 \\
\hline
\end{tabular}

the whole image region. Before we present our extensive feature study comparing several traditional texture descriptors and their erosion band versions for the problem of leaf spot classification, we briefly introduce the used classifier.

\section{Classification}

To predict the disease per region we train a one-vs-one multi-class support vector machine (SVM) using the radial basis function (RBF) kernel

$$
k\left(x, x^{\prime}\right)=\exp \left(-\frac{\left\|x-x^{\prime}\right\|^{2}}{2 \sigma^{2}}\right)=\exp \left(\gamma\left\|x-x^{\prime}\right\|^{2}\right),
$$

where $\sigma$ is the kernel width and $\gamma=-\frac{1}{2 \sigma^{2}}$. For all experiments we learn the SVM-cost parameter $c$ and the kernel parameter $\gamma$ via 10 -fold cross validation on the respective training splits. The considered parameter ranges are $c \in\left\{2^{-3}, 2^{-2}, \ldots, 2^{6}\right\}$ and $\gamma \in\{0.01,0.05, \ldots 5.0,10.0\}$. We train the classifier on 6 classes, where 5 are leaf spots caused by the previously introduced pathogens Cercospora beticola (cerc), Ramularia beticola (ram), Pseudomonas syringae ( $p s e u$ ), Uromyces betae (rust), and Phoma betae (phom). To handle regions extracted by the region detector not belonging to one of these classes we consider a sixth class non-infected ( $n$-inf). Example regions of this class could be healthy leaf parts, such as reflections or leaf veins, dirt on the leave, holes, or earth on the ground. These regions occur as we apply a simple and efficient region detector being feasible on the smart phone. To analyse disease detection rates, we therefore also evaluate a classification task, where we consider the classes inf and $n$-inf, where inf subsumes all regions labeled as being caused by one of the considered diseases. For this binary task we also use a SVM with the same kernel settings as described for the multi-class problem.

\section{EXPERIMENTAL EVALUATION}

In order to determine a small but meaningful feature ensemble for robust leaf spot classification, we performed an extensive feature study extending previously suggested descriptors [2], [3]. First, we evaluate each combination of statistical texture feature and input value introduced in Section II-B on the whole region and further on the erosion bands as described in Section II-C. Then, we create a feature ensemble of the best performing but most diverse feature/value combinations from the first evaluation step. This feature ensemble, called erosion feature ensemble, is then evaluated on a comprehensive dataset of 2957 regions extracted from 495 cell phone images.

\section{A. Datasets}

First, we introduce two datasets used for feature selection and evaluation. The first one, called STUDY, is a subset of all available regions extracted from 495 cell phone camera images applying the region detector described above. It was derived by manually selecting a balanced number of regions 
TABLE II. AVERAGE ACCURACIES (\%) OBTAINED BY 20-FOLD CROSS VALIDATION ON THE DATASET STUDY. MEMBERSHIP IN THE CANDIDATE FEATURE SET FOR THE EROSION FEATURE ENSEMBLE DESCRIBED IN SECTION III-C IS INDICATED IN BOLD.

\begin{tabular}{|c|c|c|c|c|c|c|c|c|c|c|c|c|c|c|}
\hline & & $\operatorname{dim}$ & $\begin{array}{l}\text { INT } \\
(1)\end{array}$ & $\begin{array}{l}\text { INT } \\
\text { LBP } \\
(1)\end{array}$ & $\begin{array}{l}\mathrm{R} \\
(1)\end{array}$ & $\begin{array}{l}\text { G } \\
(1)\end{array}$ & $\begin{array}{l}\mathrm{B} \\
(1)\end{array}$ & $\begin{array}{l}\text { RG } \\
(2)\end{array}$ & $\begin{array}{l}\mathrm{RB} \\
(2)\end{array}$ & $\begin{array}{l}\text { GB } \\
(2)\end{array}$ & $\begin{array}{l}\text { RGB } \\
(3)\end{array}$ & $\begin{array}{c}\mathrm{G}_{\mathrm{mag}} \\
(1)\end{array}$ & $\begin{array}{l}\mathrm{G}_{\text {dir }} \\
(1)\end{array}$ & $\begin{array}{l}\mathrm{G}_{\mathrm{mag}} \\
\text { LBP } \\
(1)\end{array}$ \\
\hline \multirow{7}{*}{$\begin{array}{l}\text { ప̃ } \\
\frac{0}{00} \\
\stackrel{0}{0} \\
\frac{0}{0} \\
\frac{\pi}{3}\end{array}$} & MEAN & (1) & 35.1 & 42.4 & 32.7 & 41.5 & 42.9 & 42.2 & 42.9 & 43.1 & 47.0 & 21.6 & 32.4 & 35.5 \\
\hline & VAR & (1) & 23.3 & 20.0 & 21.4 & 30.3 & 23.6 & 36.8 & 35.7 & 34.4 & 43.5 & 30.5 & 25.7 & 19.6 \\
\hline & MEAN \& VAR & $(2)$ & 37.5 & 42.0 & 30.6 & 37.1 & 47.9 & 44.1 & 49.6 & 46.9 & 54.3 & 41.2 & 33.4 & 36.1 \\
\hline & ENTROPY & (1) & 35.6 & 43.3 & 33.3 & 34.7 & 30.3 & 35.3 & 36.1 & 45.3 & 44.5 & 34.8 & 49.0 & 44.2 \\
\hline & $\begin{array}{l}\text { TEXTURE } \\
(d=1)\end{array}$ & $(7)$ & 57.4 & 52.8 & 59.3 & 56.7 & 58.8 & 65.9 & 65.5 & 64.4 & 68.2 & 51.9 & 48.7 & 48.7 \\
\hline & $\begin{array}{l}\text { TEXTURE } \\
(d=3)\end{array}$ & $(7)$ & 50.0 & 48.4 & 51.2 & 50.3 & 48.5 & 62.8 & 57.0 & 54.5 & 62.4 & 48.2 & 49.3 & 45.8 \\
\hline & $\begin{array}{l}\text { TEXTURE } \\
(d=5)\end{array}$ & $(7)$ & 49.5 & 50.8 & 48.9 & 43.5 & 46.9 & 57.7 & 54.6 & 52.6 & 57.0 & 49.3 & 46.2 & 41.2 \\
\hline \multirow{7}{*}{$\begin{array}{l}\tilde{\theta} \\
\tilde{E} \\
\tilde{\Xi} \\
\frac{\tilde{0}}{0} \\
\frac{0}{0}\end{array}$} & MEAN & $(4)$ & 54.5 & 50.5 & 49.2 & 54.6 & 52.6 & 54.3 & 53.2 & 56.0 & 54.6 & 42.8 & 30.7 & 43.9 \\
\hline & VAR & (4) & 45.1 & 29.0 & 42.5 & 45.1 & 45.5 & 52.6 & 50.7 & 44.5 & 52.3 & 36.7 & 38.2 & 32.8 \\
\hline & MEAN \& VAR & $(8)$ & 58.7 & 51.0 & 56.6 & 60.1 & 55.4 & 60.8 & 59.0 & 60.4 & 61.7 & 48.3 & 37.1 & 42.8 \\
\hline & ENTROPY & $(4)$ & 43.2 & 54.0 & 44.5 & 42.8 & 47.2 & 47.3 & 50.0 & 50.3 & 55.3 & 47.2 & 43.6 & 52.9 \\
\hline & $\begin{array}{l}\text { TEXTURE } \\
(d=1)\end{array}$ & $(28)$ & 67.7 & 55.4 & 69.0 & 65.8 & 64.8 & 71.8 & 68.8 & 71.3 & 71.9 & 48.9 & 51.4 & 53.7 \\
\hline & $\begin{array}{l}\text { TEXTURE } \\
(d=3)\end{array}$ & (28) & 62.4 & 54.4 & 67.0 & 67.1 & 60.7 & 69.1 & 66.8 & 63.8 & 67.7 & 47.2 & 51.4 & 44.2 \\
\hline & $\begin{array}{l}\text { TEXTURE } \\
(d=5)\end{array}$ & $(28)$ & 63.8 & 57.8 & 66.2 & 65.5 & 62.5 & 69.1 & 68.8 & 64.2 & 70.9 & 47.2 & 48.0 & 46.6 \\
\hline
\end{tabular}

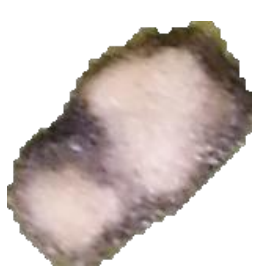

(a) Pseudomonas

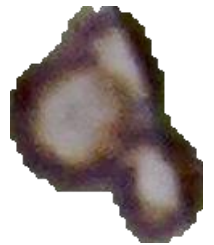

(b) Cercospora

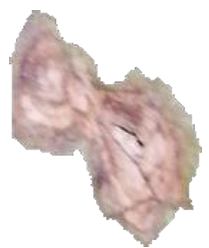

(c) Ramularia
Fig. 5. Example regions with coadunate spots of three disease symptoms.

for each class and considering regions consisting of one single leaf spot only as illustrated in Figure 3(a). This dataset is used in our feature study and hence, can be seen as training dataset for the feature selection process. The second dataset, FULL, is composed of all 2957 extracted regions and it also contains regions consisting of several coadunate leaf spots as illustrated in Figure 5. This dataset also shows high class imbalance as we did not meet many beet plants infected by Rust and Phoma during data acquisition. The class frequencies of both datasets are listed in Table I.

\section{B. Feature Study}

Before we describe the feature ensemble construction, we discuss the results of our feature study on STUDY. Feature performance is evaluated by running C-SVM classification as described in Section II-D using libSVM (http://www.csie.ntu. edu.tw/ cjlin/libsvm/) on 20 randomly generated but fixed data splits. Average accuracies for all feature/value combinations are shown in Table II.

First, we observe that the performance is universally improved by computing the texture features on the erosion bands instead of on the whole regions. This result proves the usefulness of our newly proposed erosion band features for leaf spot classification. Further, we see that LBP images of intensities (INT) and gradient magnitudes $\left(\mathrm{G}_{\text {mag }}\right)$ can improve the performance of simple statistics, such as MEAN and ENTROPY confirming results presented in [5]. In general, second- order statistics, denoted by TEXTURE, lead to better performance compared to simple first-order statistics. Considering distances $d>1$, however, did decrease accuracy, leading to the conclusion that for leaf spot classification in our scenario the co-occurrence of neighbouring pixel pairs is most informative about the disease. Note, that second-order texture descriptors as well as erosion band features are of higher dimensionality. The dimensionality of each feature/value pair can be computed as the product of feature and value dimensionality indicated in the second row and third column of Table II. Note that using all feature/value combinations results in a 960-dimensional input space leading to a less efficient classification cascade.

\section{Erosion Feature Ensemble}

To build a small, yet powerful and diverse feature ensemble from these results, we consider the following grouping of feature/value pairs: first, we contrast computation on the whole region versus on erosion bands; second, we divide the values in intensity-based, color-based and gradient-based; and then we use the natural distinction of simple, i.e. first-order, versus second-order statistics. This leads to 12 feature/value groups including erosion and non-erosion based descriptors. Now, we consider the best performing pair in each group to be a candidate for our feature ensemble. The performances of these candidates are marked bold in Table II; the groups are indicated by blocks. The final feature ensemble is derived from forward selection followed by backward elimination on the candidate set. With this approach we traced the combination of erosion band TEXTURE descriptors with $d=1$ on the three color channels (RGB) and on the gradient magnitude LBPS $\left(G_{\text {mag }}\right.$ LBP $)$. This best performing feature ensemble, named erosion feature ensemble (EFE), has 112 dimensions and its classification accuracy is $75.2 \%$ on the dataset STUDY.

\section{Results}

We performed a 20-fold cross validation to evaluate EFE on the full dataset covering all extracted regions from the available 495 cell phone images. We study two tasks, disease detection 
TABLE III. AVERAGE ACCURACIES AND STANDARD DEVIATIONS IN \% (AVERAGE PREDICTION TIME IN SECONDS) OF THE EROSION FEATURE ENSEMBLE (EFE), ALL FEATURES (ALL), MOST FREQUENT CLASS (MFC) AND RANDOM (RAND) FOR DISEASE DETECTION AND CLASSIFICATION.

\begin{tabular}{|c|c|c|c|c|c|c|c|c|}
\hline & \multicolumn{3}{|c|}{ disease detection } & \multirow[b]{2}{*}{ RAND } & \multicolumn{4}{|c|}{ disease classification } \\
\hline & EFE & ALL & MFC & & EFE & ALL & MFC & RAND \\
\hline STUDY & $93.9 \pm 8.4\left(0.00^{\prime \prime}\right)$ & $92.5 \pm 7.0\left(0.00^{\prime \prime}\right)$ & 81.3 & 50.0 & $75.2 \pm 8.6\left(0.00^{\prime \prime}\right)$ & $77.0 \pm 11.8\left(0.01^{\prime \prime}\right)$ & 19.3 & 16.7 \\
\hline FULL & $93.3 \pm 1.7\left(0.02^{\prime \prime}\right)$ & $95.4 \pm 2.1\left(0.15^{\prime \prime}\right)$ & 62.6 & 50.0 & $83.8 \pm 3.3\left(0.04^{\prime \prime}\right)$ & $87.6 \pm 2.5\left(0.38^{\prime \prime}\right)$ & 37.4 & 16.7 \\
\hline
\end{tabular}

to predict whether a leaf spot is caused by an infection or not and disease classification, where we predict one out of six classes (cerc, ram, pseu, rust, phom, and n-inf) per region.

For disease detection we consider two classes, inf and $n$ inf, where the former subsumes all infected regions. Using EFE we achieve $93.3 \%$ accuracy on FULL (93.9\% on STUDY), cf. Table III. When detecting diseases we are especially interested in a high recall. We detect $95.4 \%$ (FULL), respectively $99.2 \%$ (STUDY), of all infected leaf spots as being caused by a disease. Without feature selection (ALL) detection accuracy is worse for STUDY and only slightly higher for FULL while requiring more computation time. These results clearly show that erosion band features are highly appropriate for the identification of leaf spots caused by fungal and bacterial diseases.

For disease classification EFE leads to an average accuracy of $83.7 \%$ whereas the use of all descriptor/value combinations (ALL) achieves $87.6 \%$ on the dataset FULL. This is a highly convincing result as the classification problem has six classes. Random class assignment results in an accuracy of $16.7 \%$ and predicting the most frequent class yields $37.5 \%$. ALL does outperform EFE, however, feature computation and prediction times increase by an order of magnitude as the dimension of the considered feature space increases from 112 to 960 . These results, summarized in Table III, confirm the power of erosion based texture descriptors for leaf spot classification and justify our proposed feature selection method. For FULL the confusion matrix to analyse the per class performance of EFE is

$$
M=\left[\begin{array}{cccccc|c}
1009 & 26 & 2 & 58 & 15 & 6 \\
39 & 891 & 0 & 94 & 28 & 14 & \text { n-inf } \\
2 & 0 & 65 & 1 & 0 & 0 & \text { cerc } \\
\text { rust } \\
44 & 64 & 5 & 335 & 17 & 3 & \text { pseu } \\
9 & 24 & 0 & 6 & 163 & 16 & \text { ram } \\
2 & 1 & 0 & 0 & 2 & 16
\end{array}\right] \text { phom }
$$

where the off-diagonals of the rows (columns) show the number of false positives (false negatives). All classes except phom have a classification accuracy above $67 \%$. The misclassified leaf spots caused by Phoma where frequently labeled as Cercospora or Ramularia. This is due to their similar appearance and the lack of sufficient training data in this class; less than $2 \%$ of the regions have the label phom. These results suggest that we can achieve even higher performance rates for this class once having access to more training data.

\section{CONCLUSIONS}

We introduced a new feature derivation scheme for leaf spot classification based on statistical texture features computed on multiple erosion bands of regions of interest extracted from cell phone images of beet leaves. In addition, we conducted an extensive feature study and systematically derived the erosion feature ensemble, a discriminative, diverse, and robust feature ensemble for leaf spot classification. Although our feature design allows for easy and efficient computation, it goes beyond the use of descriptors simply computed on the whole extracted regions as previously used for plant disease classification. In summary, we presented an efficient holistic approach to classify leaf spots extracted from cell phone images of beet leaves as being caused by one out of five different diseases.

The success of erosion-based texture features suggests to apply similar techniques to disease classification of other plants, such as wheat or cotton in the future. Further, we plan to integrate the developed erosion band features in an automatic consultation system reliably helping farmers in the field to assess the kind and amount of fungicides needed for pest control. This will result in reduced costs and environmental burden due to a more targeted treatment.

\section{ACKNOWLEDGMENT}

This work is supported by the Federal Ministry of Food, Agriculture and Consumer Protection (BMELV) based on a decision of the German Federal Office for Agriculture and Food (BLE) under the innovation support programme; grant nr. "2815411310".

\section{REFERENCES}

[1] G. Agrios, Plant Pathology, 4th ed. Academic Press, 1997.

[2] A. Camargo and J. Smith, "Image pattern classification for the identification of disease causing agents in plants," Computers and Electronics in Agriculture, vol. 66, no. 2, pp. 121-125, 2009.

[3] H. Al-Hiary, S. Bani-Ahmad, M. Reyalat, M. Braik, and Z. Alrahamneh, "Fast and accurate detection and classification of plant diseases," International Journal of Computer Applications, vol. 17, no. 1, pp. 3138, 2011.

[4] R. M. Haralick, K. Shanmugam, and Dinstein, "Textural Features for Image Classification," Systems, Man and Cybernetics, IEEE Transactions on, vol. SMC-3, no. 6, pp. 610-621, 1973.

[5] T. Ojala, M. Pietikäinen, and T. Mäenpää, "Multiresolution Grayscale and Rotation Invariant Texture Classification with Local Binary Patterns," IEEE Trans. PAMI, vol. 24, no. 7, 2002.

[6] R. Gonzales and R. Woods, Digital Image Processing, 3rd ed. Pearson Prentice Hall, 2008.

[7] C. Bauckhage, "Tree-Based Signatures for Shape Classification," in Proceedings of the International Conference on Image Processing (ICIP-06), 2006, pp. 2105-2108.

[8] Y. Dupain, T. Kamae, and M. Mendes-France, "Can One Measure the Temperature of a Curve," Archive for Rational Mechanics and Analysis, vol. 94, no. 2, pp. 155-163, 1986.

[9] Y. Ebrahim, M. Ahmed, S. Chau, and W. Abdelsalam, "An Efficient Shape Representation and Description Technique," in Proceedings of the International Conference on Image Processing (ICIP-07), 2007, pp. 441-444.

[10] X. Huang, S. Z. Li, and Y. Wang, "Shape Localization Based on Statistical Method Using Extended Local Binary Pattern," in Third International Conference on Image and Graphics (ICIG-04), 2004, pp. 184-187.

[11] D. Maturana, D. Mery, and A. Soto, "Face Recognition with Local Binary Patterns, Spatial Pyramid Histograms and Naive Bayes Nearest Neighbor Classification," in 2009 International Conference of the Chilean Computer Science Society (SCCC-09), 2009, pp. 125-132.

[12] E. Vazquez, X. Yang, and G. G. Slabaugh, "Erosion band signatures for spatial extraction of features," Machine Vision Applications, vol. 24, no. 4, pp. 695-705, 2013. 